\title{
Chest pain during exercise as first manifestation of Friedreich's ataxia
}

\author{
Pere Ferrés-Sánchez, Maite Subirana-Domènech, Miquel Torner-Soler
}

\begin{abstract}
Cardiac function is affected in up to $90 \%$ of patients with Friedreich's ataxia, the most common spinocerebellar degenerative disease. Friedreich's ataxia typically causes motor abnormalities of the extremities, mainly impairing walking and the coordination of the legs and arms. The myocardium is affected at a later stage of the disease. The extent and timing of myocardial involvement determines the clinical course. Some patients have no cardiac symptoms and cardiac involvement can be established only by electrocardiographic or echocardiographic examination. In addition some pathological studies have found evidence of coronary abnormalities, mainly in the small vessels. There are no reports that such lesions cause angina.
\end{abstract}

In a 16 year old patient chest pain on exercise had been the presenting symptom of Friedreich's ataxia at the age of 9. Considerable alterations in ventricular repolarisation on the electrocardiogram suggested a congenital coronary abnormality or hypertrophic myocardiopathy. The results of a Doppler echocardiography, Holter monitoring, and a haemodynamic study with coronary arteriography were all normal. An exercise test when the boy was 13 indicated significant changes in ventricular repolarisation. Myocardial scintigraphy ( ${ }^{99 m}$ Tc-MIBI) at that time, however, was normal. He improved slightly when he was treated with verapamil. When he was 15 neurological symptoms developed and Friedreich's ataxia was diagnosed. Typical angina during exercise seems to have been the first symptom of Friedreich's ataxia.

Department of Cardiology, Hospital de la Santa Creu i Sant Pau, $S$ Antoni M: Claret, 167

08025 Barcelona

P Ferrés-Sánchez

$M$ Subirana-Domènech

$M$ Torner-Soler

Correspondence to:

Dr P Ferrés-Sánchez, C/Barcelona 27,

08301-Mataro, Spain.

Accepted for publication 27 March 1995
(Br Heart f 1995;74:464-467)

Keywords: Friedreich's ataxia; chest pain

Cardiac involvement in Friedreich's ataxia

Friedreich's ataxia is the most common degenerative spinocerebellar disease. It is caused by a recessive autosomal gene on chromosome $9 .{ }^{1}$
Cardiac involvement is seen in $50-90 \%$ of cases. ${ }^{2}$ Patients can remain symptom free for several years and the diagnosis is established by means of an electrocardiogram (ECG) or an echocardiogram or both. There are ECG alterations in $80-100 \%$ of patients ${ }^{13-6}$ and echocardiographic abnormalities in $50-86 \% .{ }^{1367}$

The most frequent cardiac pathology is hypertrophic myocardiopathy, ${ }^{2-8}$ which is usually concentric, with preserved systolic function. Nevertheless, in some cases the septum is predominantly affected and there can be a subaortic gradient. A less common finding with a much poorer prognosis, is dilatated myocardiopathy with reduced systolic function. This is associated with tachyarrhythmias, development of congestive cardiac failure, and sudden death. It is not known whether these different forms are merely different stages of the disease or different effects. ${ }^{9}$

The pathogenesis of cardiac lesions still remains unknown. James et al ${ }^{10}$ in a postmortem examination of three patients with Friedreich's ataxia who all died from congestive cardiac insufficiency (congestive heart failure) and atrial arrhythmias (one of these cases had already been reported ${ }^{11}$ ) suggested the possibility of a multifactorial pathogenesis. Their mosaic concept of pathogenesis involved ischaemic factors affecting both small and large coronary arteries; neurological factors affecting nerves and lymph nodes (cardioneuropathy); and myopathic factors with focal fibrosis and cellular lesions, especially at the level of the myocytes (cardiomyopathy).

Coronary involvement, especially of small vessels, has been described in several studies but in most its possible functional consequences were not evaluated. ${ }^{10-13}$ When Hewer reviewed necropsy specimens from 27 cases of Friedreich's ataxia, he found lesions in some small coronary arteries. ${ }^{13}$ However, he did not regard these lesions as important, because so often arteries (about $9 \%$ ) were affected. $\mathrm{He}$ did not think that these lesions could be responsible for the extreme muscle fibrosis. Hewer thought that the arterial narrrowing was secondary to degeneration of the cardiac muscle. We believe that the number of samples he studied represented only a very small part of the coronary arterial tree, too small a part to assess the overall involvement of the 
Figure 1 (Left) Repolarisation abnormalities in the electrocardiogram at rest with negative $T$ waves in leads I, II, III and VF and all precordial leads. (Right) Significant ST segment elevation in leads V1-4 during an exercise test
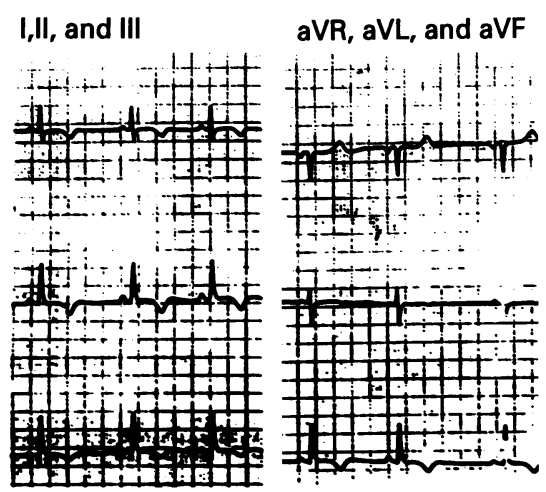

V1,2, and 3

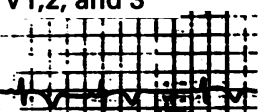

V4,5, and 6

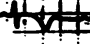

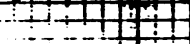

$-1+1+1+1$
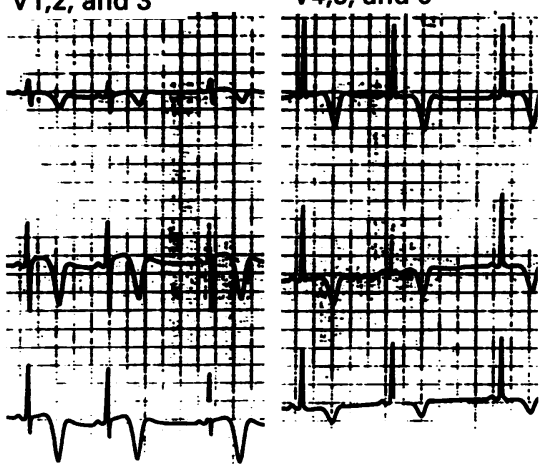
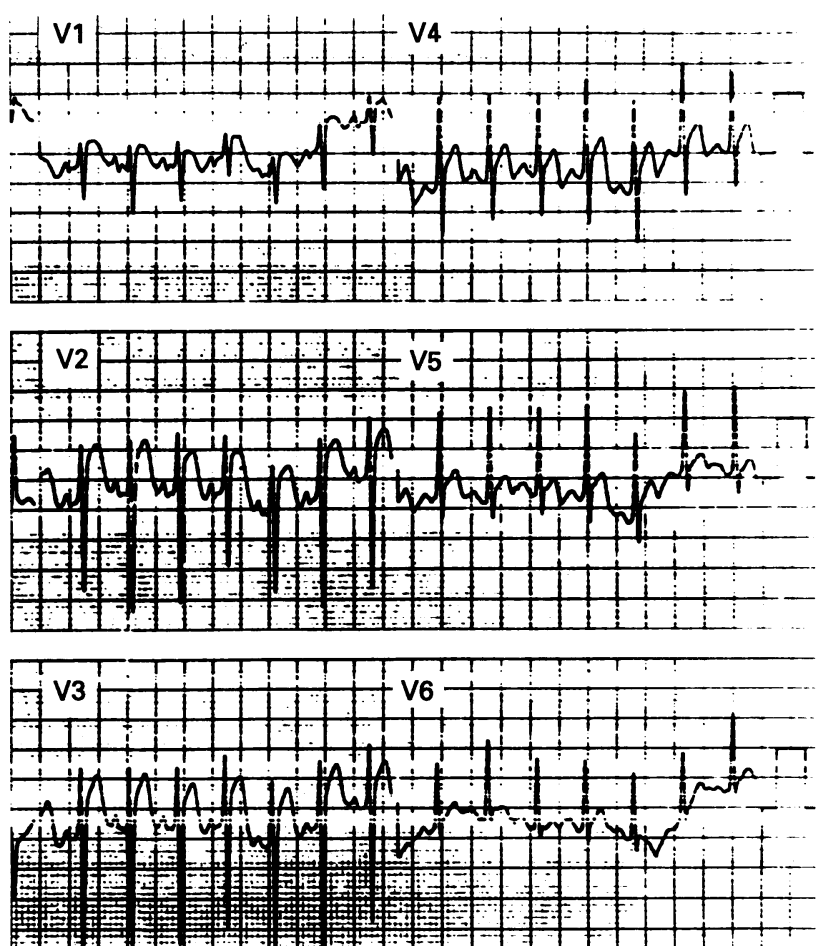

m

$\stackrel{T}{\mathbb{D}}$

coronary arterial tree, especially as any coronary abnormality is likely to have been focal. Nor is there any evidence that these lesions are secondary to the actual myocardial pathology. Russell ${ }^{14}$ found lesions causing stenosis of the large arteries in two of his four cases, and Boyer et $a l^{15}$ found atheromatous lesions in one (a 25 year old woman) of two necropsy cases. Such anatomical lesions may not be the sole cause of coronary ischaemia, however, both arterial tone and vasomotor reactivity are possible causes of ischaemic myocardiopathy. Coronary spasm can affect both factors. Margalith et al described a case of Friedreich's ataxia in which coronary spasm was detected. ${ }^{16}$

\section{Case report}

We present the case of a 16 year old boy with an unremarkable personal and family history in whom angina during exercise developed when he was 9. A resting electrocardiogram subsequently showed abnormal ventricular repolarisation, with negative $T$ waves in all leads (fig 1A). A standard echo-Doppler study was normal. Holter monitoring for $24 \mathrm{~h}$ showed no significant changes in ventricular repolarisation at the high cardiac rates at which the chest pain had occurred. While the patient was undergoing a standard Bruce protocol exercise testing he complained of precordial pain which made it necessary to stop the test. There were slight changes in the ST segment. Myocardial scintigraphy (thallium-201) showed signs of posterobasal and inferior hypoperfusion without redistribution. In view of this finding, a haemodynamic study with coronary arteriography was performed. This did not show any coronary lesions and confirmed that left ventricular systolic function was normal.
Given the absence of morphological abnormalities, the case was classified as "thoracic pain during physical exercise" with no clear evidence of myocardial ischaemia. The patient was instructed to have regular cardiological examinations. The symptoms continued but the patient learnt to stop exercising before becoming ill.

When he was 13 he was referred to our hospital. The clinical tests were repeated. The results were identical with those obtained earlier (fig 2), except for the exercise test, which showed that repolarisation had become more abnormal (fig 1B). Myocardial scintigraphy with ${ }^{99 m}$ Tc-labelled methoxyisobutyl isonitrile (MIBI), however, was normal. Treatment with nifedipine ( $30 \mathrm{mg} /$ day) was started, without significant improvement. Verapamil was then tried $(240 \mathrm{mg} /$ day $)$. This delayed the onset of thoracic pain on exercise, as confirmed in repeat standard Bruce protocol exercise testing.

During the physical examination kyphoscoliosis was found and he consulted an orthopaedic surgeon. At consultation, Friedreich's ataxia was suspected. Over the next year incoordination of the legs became progressively evident.

When he was 16 a new cardiac ultrasound study showed a slightly hypertrophied, nondilatated left ventricle (septum $12 \mathrm{~mm}$, posterior wall $11 \mathrm{~mm}$ ) with normal systolic function (fig 3).

\section{Comments}

We believe that there are enough reported studies to confirm that coronary involvement is an integral part of the cardiomyopathy associated with Friedreich's ataxia. Our patient seems to be an example of such 
Figure 2 Normal

echocardiographic study when the patient was 13 years old

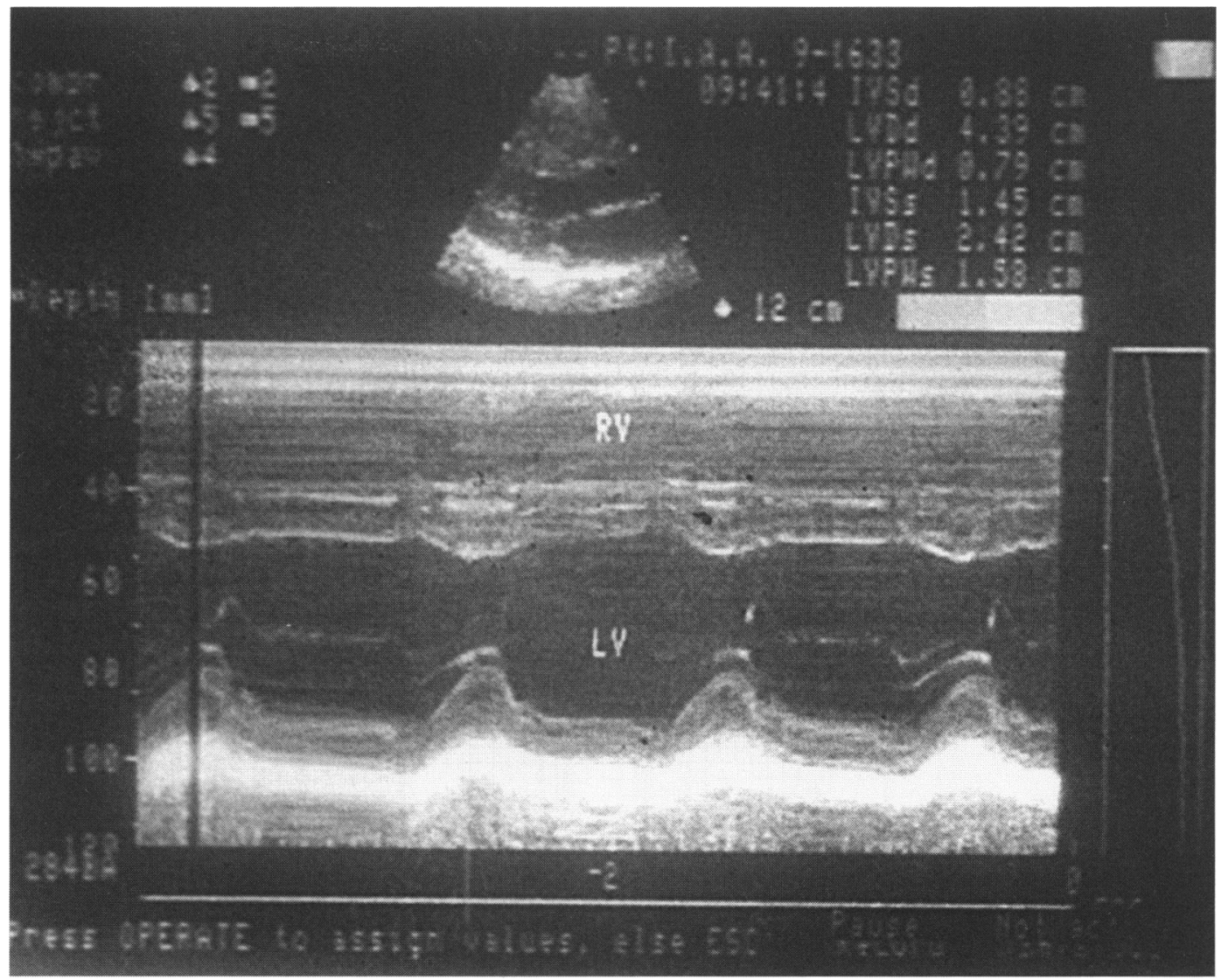

involvement. We cannot, however, rule out a vasospastic factor in our patient. No biopsy was carried out to assess whether there were lesions in the small coronary vessels, but in view of the published data and the clinical history of the patient, we think we have

sufficient evidence to regard coronary ischaemia as the cause of thoracic pain in our patient.

Angina is a rare symptom in children and adolescents and we have not been able to find any other cases that resemble our patient.
Figure 3 Echocardiogram showing slight hypertrophy of the left ventricle when the patient was 16 years old

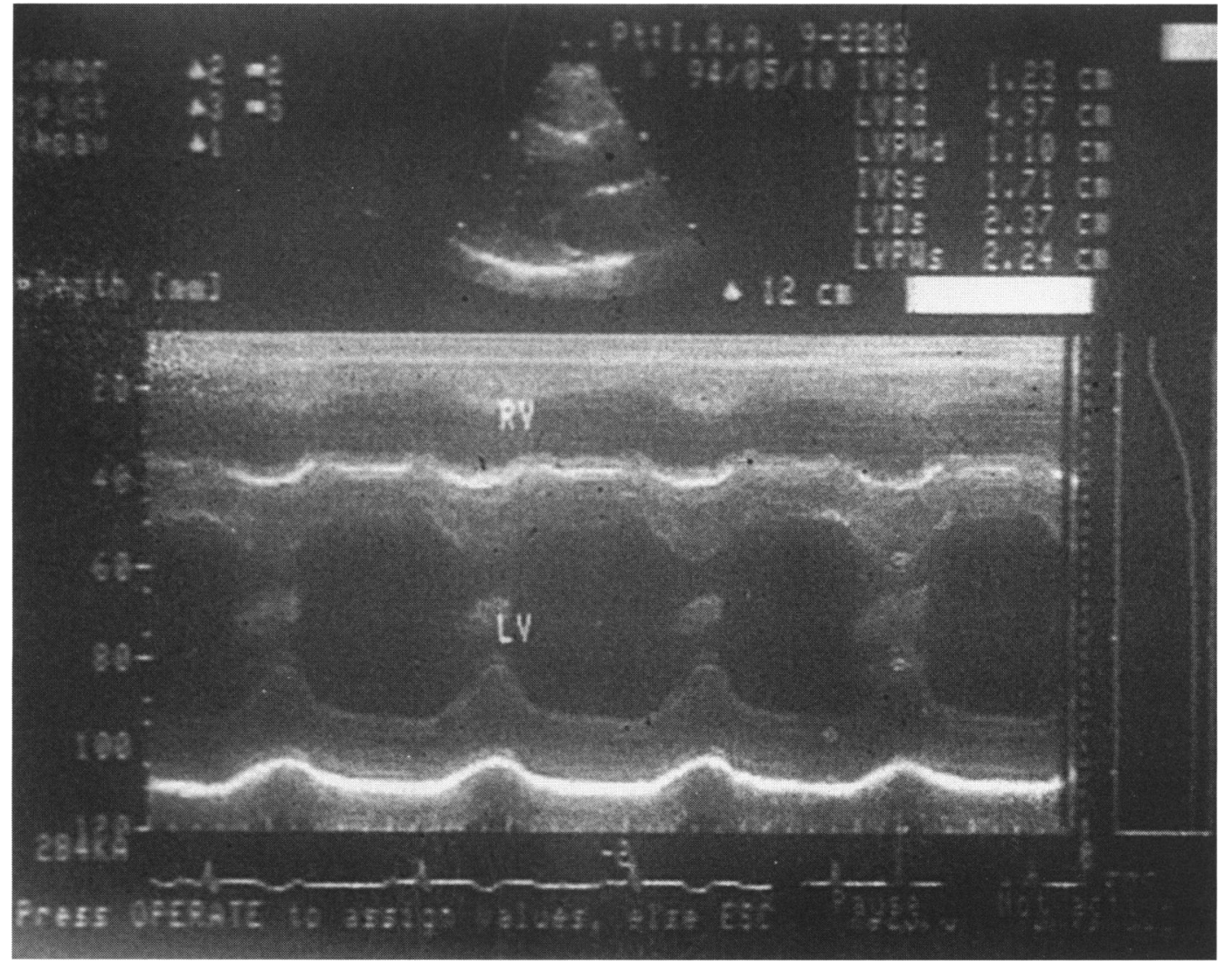


1 Muller-Felber W, Rossmanith T, Spes C, Chamberlain S, Pongratz D, Deufel T. The clinical spectrum of Friedreich's ataxia in German families showing linkage to the FRDA locus on chromosome 9. Clin Investig 1993;71: 109-14.

2 Unverferth DV, Schmidt WR, Baker PB, Wooley CF. Morphologic and functional characteristics of the heart in Friedreich's ataxia. $A m \mathcal{F} M e d$ 1987;82:5-10.

3 Casazza F, Ferrari F, Piccone U, Maggliolini S, Capozi A, Morpurgo M. Progression of cardiopathology in Morpurgo M. Progression of cardiopathology in 1990;35:423-31.

4 Salih MA, Ahlsten G, Stalberg E, et al. Friedreich's ataxia in 13 children: presentation and evolution with neurophysiologic, electrocardiographic and echocardiographic features. $f$ Child Neurol 1990 ; 5 .321-6.

5 Giunta A, Maione S, Biagini R, Filla A, De Michele G, Campanella $G$. Noninvasive assessment of systolic and diastolic function in 50 patients with Friedreich's ataxia. Cardiology 1988;75:321-7.

6 Alboliras ET, Shub C, Gomez MR, et al. Spectrum of cardiac involvement in Friedreich's ataxia: Clinical, electrocardiographic and echocardiographic observations. $A m \mathcal{F}$ Cardiol 1986;58:518-24.

7 Illarioshkin SN, Borisendo, VV, Ivanova-Smolenskala IA. Cardiac changes in hereditary spinocerebellar degenera- tions. Ter Arkh 1990;62:88-92.

8 Morvan D, Komajda M, Do Doan L, et al. Cardiomyopathy in Friedreich's ataxia: a Doppler-echocardiographic study. Eur Heart f 1992;13:1393-8.

9 Child JS, Perloff JK, Bach PM, et al. Cardiac involvement in Friedreich's ataxia: a clinical study of 75 patients. $7 \mathrm{Am}$ Coll Cardiol 1986;7:1370-8.

10 James TN, Cobbs BW, Coghlan HC, McCoy WC, Fisch C. Coronary disease, cardioneuropathy, and conduction system abnormalities in the cardiomyopathy of Friedreich's ataxia. Br Heart f 1987;57:446-57.

11 James TN, Fisch C. Observations on the cardiovascular involvement in Friedreich's ataxia. Am Heart $\mathcal{f} 1963$; 66:164-75.

12 Nadas AS, Alimurung MM, Sieracki LA. Cardiac manifestations of Friedreich's ataxia. $N$ Engl $\mathcal{F}$ Med 1951;244 239-44.

13 Hewer RL. The heart in Friedreich's ataxia. Br Heart $f$ 1969;31:5-14.

14 Russell DS. Myocarditis in Friedreich's ataxia. $f$ Pathol 1946;53:739-48.

15 Boyer SH, Chisholm AW, McKusick VA. Cardiac aspects of Friedreich's ataxia. Circulation 1962;25:493-505.

16 Margalith D, Dunn HG, Carter JE, Wright JM Friedreich's ataxia with Dysautonomia and labile hypertension. Can $\mathcal{F}$ Neurol Sci 1984;11:73-7. 